\title{
Artigo/Article
}

\section{Impacto do tratamento antirretroviral na ocorrência de macrocitose em pacientes com HIV/AIDS do município de Maringá, Estado do Paraná}

\author{
Impact of antiretroviral therapy on occurrences of macrocytosis in patients with HIV/AIDS \\ in Maringá, State of Paraná
}

\section{Odete Correia Antunes de Oliveira ${ }^{1}$, Ramon Antunes de Oliveira $^{2}$ e Lenice do Rosário de Souza ${ }^{3}$}

\section{RESUMO}

Introdução: AIDS é uma doença causada pelo HIV que compromete o sistema imune do organismo. $\mathrm{O}$ advento da terapia antirretroviral (TARV) altamente eficaz promoveu melhora substancial do prognóstico da doença e da qualidade de vida dos pacientes com HIV/AIDS. Durante seu tratamento prolongado, notam-se algumas alterações hematológicas, dentre elas, anemia e macrocitose, bem como carências de micronutrientes, tais como, de vitamina B12 e ácido fólico. O objetivo do presente trabalho é relacionar a macrocitose e anemia ao uso de TARV, ou à deficiência de vitamina B12 ou de ácido fólico. Métodos: Foram avaliados 110 pacientes HIV positivos, comparando-se aqueles em uso de TARV com zidovudina (AZT) (grupo 1), TARV sem AZT (grupo 2) ou sem uso de TARV (grupo 3). Resultados: Os pacientes dos três grupos não apresentaram diferenças estatísticas significativas quanto aos níveis de hemoglobina $(\mathrm{p}=0,584)$ e de ácido fólico $(\mathrm{p}=0,956)$. Os pacientes do grupo $1(\mathrm{G} 1)$ apresentaram volume corpuscular médio (VCM) aumentado quando comparado ao grupo 3 (G3) (p < 0,05), bem como do grupo 2 (G2) em relação ao G3 ( $p<0,001)$. As dosagens de vitamina B12 do G1 e $\mathrm{G} 3$ foram menores do que as encontradas pelo $\mathrm{G} 2(\mathrm{p}=0,008)$. Conclusões: Conclui-se que os indivíduos em uso de TARV apresentaram macrocitose, embora não pudesse ser relacionada ao tipo de TARV ou a deficiência de vitamina B12. Entretanto, a deficiência de ácido fólico não esteve relacionada ao uso de TARV e nem à macrocitose.

Palavras-chaves: AIDS. Vitamina B12. Ácido fólico. Anemia macrocítica. Terapia antirretroviral de alta atividade.

\begin{abstract}
Introduction: AIDS is a disease caused by HIV that compromises the organism's immune system. The advent of highly active antiretroviral therapy (HAART) has promoted substantial improvement in the prognosis for this disease and in HIV/AIDS patients' quality of life. During prolonged treatment, certain hematological disorders are observed, such as anemia and macrocytosis, as well as deficiencies of micronutrient such as vitamin B12 and folic acid. The objective of this study was to correlate the presence of macrocytosis and anemia with HAART use or vitamin B12 and folic acid deficiencies. Methods: 110 HIV-positive patients were included, in three groups: HAART use with zidovudine (AZT) (group 1), HAART use without AZT (group 2) and no HAART (group 3). Results: None of the patients in any of the three groups presented statistically significant differences relating to hemoglobin level $(\mathrm{p}=0.584)$ or folic acid level $(\mathrm{p}=0.956)$. Group $1(\mathrm{G} 1)$ had a higher mean corpuscular volume (MCV) than G3 $(\mathrm{p}<0.05)$, and group $2(\mathrm{G} 2)$ had a higher volume than group $3(\mathrm{G} 3)(\mathrm{p}<0.001)$. Vitamin B12 levels in G1 and G3 were smaller than those in G2 $(p=0.008)$. Conclusions: It was concluded that patients undergoing HAART treatment presented macrocytosis, even though this could not be correlated with the type of HAART or with vitamin B12 deficiency. However, folic acid deficiency was unrelated to either HAART or macrocytosis.
\end{abstract}

Keywords: AIDS. Vitamin B12. Folic acid. Macrocytic anemia. Highly active antiretroviral therapy.

1. Departamento de Doenças Tropicais e Diagnóstico por Imagem, Faculdade de Medicina de Botucatu, Universidade Estadual Paulista, Botucatu, SP. 2. Faculdade de Medicina, Universidade Federal do Paraná, Curitiba, PR. 3. Departamento de Doenças Tropicais e Diagnóstico por Imagem, Faculdade de Medicina de Botucatu, Universidade Estadual Paulista, Botucatu, SP.

Endereço para correspondência: Dra. Odete Correia Antunes de Oliveira. Dept ${ }^{\circ}$ Doenças Tropicais e Diagnóstico por Imagem/FMB/UNESP. Distrito de Rubião Júnior s/n, 18618-970 Botucatu, SP. Tel: 55 14 3811-6379; Fax: 5514 3815-9898

e-mail: odeteco@hotmail.com

Recebido para publicação em 27/06/2010

Aceito em 06/10/2010

\section{INTRODUÇÃO}

AIDS é uma doença que representa um dos grandes problemas de saúde da atualidade. No Brasil, de 1980 a junho de 2008, foram notificados 506.499 casos de AIDS. O total de óbitos acumulados até 2007 é de $205.409^{1}$. A incidência da epidemia na população foi de 17,5/100.000 em 2006, com predominância do sexo masculino de $1,5 / 1^{1}$. No município de Maringá-PR, foram notificados 37 novos casos de AIDS em 2007, sendo que o sexo masculino corresponde a $67,5 \%$ dos casos $^{2}$.

A introdução da terapia antirretroviral (TARV) propiciou grandes avanços no tratamento da AIDS, com dramática redução da morbidade e da mortalidade associadas à doença ${ }^{3}$. Os efeitos colaterais têm sido um dos importantes fatores da não adesão à TARV. Um exemplo de efeitos imediatos comum a todas as classes são os decorrentes de intolerância gástrica caracterizada por náuseas, vômitos e dor abdominal. Eventos adversos comumente observados após o uso de inibidores de transcriptase reversa análogos de nucleosídeos (ITRN) são: astenia, cefaléia ou insônia. O AZT, um exemplo entre os ITRN, está associado com mielotoxicidade, principalmente anemia, pancitopenia e macrocitose $\mathrm{e}^{4}$.

A infecção pelo HIV pode apresentar uma ampla gama de anormalidades hematológicas, como o efeito supressivo do próprio HIV, hematopoiese ineficaz, doenças infiltrativas da medula óssea, consumo periférico secundário à esplenomegalia ou desregulação imune e deficiências nutricionais ${ }^{5}$.

A anemia é comum em indivíduos com infecção pelo HIV assintomáticos ocorrendo em, aproximadamente, $30 \%$ dos casos, e taxas extremamente altas são encontradas naqueles com clínica de AIDS, chegando a $80 \%{ }^{6}$. Ela decorre, frequentemente, de causas multifatoriais.

Os tipos mais comuns de anemia encontrados na prática clínica são as microcíticas e hipocrômicas. Dentre elas, destacam-se a deficiência de ferro levando a anemia ferropriva, e anemia secundária a doenças crônicas ${ }^{7}$. 
A anemia megaloblástica é outra variante que se apresenta normocrômica e macrocítica, em decorrência da deficiência ou alteração no metabolismo da vitamina B12 e/ou do ácido fólico ${ }^{8}$.

Os níveis de folato circulantes, por serem fortemente influenciados pela ingestão recente de alimentos, não são confiáveis como índice de armazenamento nos tecidos. Desta forma, os níveis de folato medidos no soro ou no plasma podem ser normais mesmo em face de sua deficiência. Inversamente, os níveis circulantes podem estar baixos muito antes do depósito em tecido ter sido esgotado?.

Embora a baixa concentração sérica de vitamina B12 tenha sido descrita em $1 / 3$ dos adultos infectados pelo HIV, a maioria destes indivíduos não tem anemia megaloblástica ${ }^{10}$.

O volume corpuscular médio (VCM) é um índice hematimétrico na medida, que permite a classificação das anemias em microcítica, normocítica e macrocítica. Considera-se macrocitose, valores de VCM maiores que $100 \mathrm{fl}^{11,12}$, entretanto valores acima de $120 \mathrm{fl}$ sugerem fortemente o diagnóstico de anemia megaloblástica ${ }^{13}$.

A macrocitose pode decorrer tanto das anemias carenciais como da deficiência de vitamina B12 ou ácido fólico, como de cirrose hepática, abuso de álcool, hipotireoidismo ou como efeitos colaterais de algumas drogas ${ }^{12}$. Drogas antirretrovirais, tais como AZT e estavudina $(\mathrm{d} 4 \mathrm{~T})$, são as que mais se relacionam à macrocitose após seu uso crônico, podendo, inclusive, ser um fator que, individualmente, demonstra adesão ao $\mathrm{AZT}^{8}$.

O objetivo deste trabalho foi avaliar a ocorrência de macrocitose em indivíduos com infecção pelo HIV ou AIDS atendidos no Programa de DST/AIDS do município de Maringá-PR e relacionálas com o uso de antirretrovirais, à deficiência de ácido fólico ou de vitamina B12.

\section{MÉTODOS}

Primeiramente, foi realizada análise retrospectiva dos 450 prontuários médicos dentre os 1.200 indivíduos cadastrados que frequentam regularmente o Serviço do Programa Municipal DST/ AIDS, do município de Maringá-PR, credenciado pelo Sistema Único de Saúde (SUS). Foram, então, estudados 110 portadores de HIV/AIDS, no período de agosto 2008 a junho de 2009, que se apresentavam maiores de 18 anos, em uso ou não de TARV, segundo indicação médica. Para estes pacientes foram registrados: sexo, idade, tempo de uso da TARV, contagem de linfócitos T CD4+, determinação da carga viral plasmática do HIV, se os pacientes faziam ou não uso de vitamina B12 ou ácido fólico, esquema atual de TARV, níveis séricos de hemoglobina e de $\mathrm{VCM}$ anteriores à pesquisa. $\mathrm{Na}$ segunda fase da coleta de dados, foram realizadas entrevistas com os pacientes. Nessa entrevista foram confirmados alguns dados que se constituíam em critérios de exclusão, tais como, uso de ácido fólico e vitamina B12, dietas vegetarianas estritas, gestantes, medicamentos para tratamento de outras infecções, de dislipidemias e neoplasias. Posteriormente, foi realizada a coleta de sangue para análise dos seguintes exames laboratoriais: dosagens de hemoglobina sérica, do volume corpuscular médio (VCM), do ácido fólico, da vitamina B12, da carga viral plasmática do HIV e linfometria T CD4+.

Constituíram-se os seguintes grupos de estudo: grupo 1 (G1): 55 pacientes infectados pelo HIV em uso de TARV com AZT; grupo 2 (G2): 23 pacientes infectados pelo HIV em uso de TARV sem AZT; grupo 3 (G3): 32 pacientes infectados pelo HIV sem uso de TARV.
Os resultados foram submetidos à análise estatística, por meio de exploração da distribuição probabilística do teste de Shapiro-Wilk, resumo descritivo, em medianas e quartis, teste de qui-quadrado para duas amostras independentes, teste de Mann-Whitney para amostras independentes, teste exato de Fisher, teste de KruskalWallis para grupos independentes, seguido do teste de Dunn para comparações múltiplas. Diferenças foram consideradas significativas se $\mathrm{p}$-valor $<0,05$.

\section{Considerações éticas}

Este estudo foi submetido à análise e aprovação do Comitê de Ética em Pesquisa da Faculdade Ingá - UNINGÁ Maringá-PR, recebendo parecer favorável no dia 29/07/2008.

\section{RESULTADOS}

A amostra foi composta por 110 portadores de infecção pelo HIV ou AIDS, dividida em três grupos, a saber: 55 no G1 (50\%), 23 no G2 (20,9\%) e 32 indivíduos no G3 (29,1\%).

Os grupos apresentaram-se pareados quanto ao gênero, com leve predominância do sexo masculino no G2 (60,9\% destes). No entanto, não houve diferença estatística significativa na comparação entre os grupos $(\mathrm{p}>0,05)$.

Os valores correspondentes às contagens de linfócitos T CD4+ não apresentaram diferença estatística significativa na comparação dos dados encontrados nos três grupos $(p=0,270)$.

A determinação da carga viral plasmática do HIV apresentou diferença estatisticamente significativa na comparação de G1 e G2 em relação a $\mathrm{G} 3$ ( $\mathrm{p}<0,001)$, ao se comparar a porcentagem de indivíduos com carga viral indetectável em cada grupo. O G1 apresentou 72,2\% e o G2 apresentou 60,9\% dos indivíduos com carga viral indetectável, frente a 3,2\% dos indivíduos do G3.

A distribuição dos indivíduos, em relação às dosagens de ácido fólico, está apresentada na Tabela 1. Na comparação entre os grupos, no entanto, não houve diferença estatística significativa em relação às dosagens de ácido fólico $(\mathrm{p}=0,956)$.

A distribuição dos indivíduos, em relação aos níveis séricos de vitamina B12 está apresentada na Tabela 2. Houve diferença estatística significativa na comparação entre G1 e G3 quando comparados ao G2 ( $p=0,008)$, e os grupos G1 e G3 não apresentaram diferença estatística entre si $(\mathrm{p}>0,05)$.

A distribuição dos indivíduos quanto aos níveis de hemoglobina, antes e no momento atual deste estudo, está mostrada na Tabela 3. Os grupos estudados, em relação aos níveis baixos de hemoglobina $(<12 \mathrm{~g} / \mathrm{dL})$ e os níveis normais de hemoglobina ou, seja, acima de

TABELA 1 - Distribuição dos 110 pacientes infectados pelo HIV, de acordo com os níveis de ácido fólico.

\begin{tabular}{|c|c|c|c|c|c|c|c|}
\hline \multirow[b]{2}{*}{ Ácido fólico $(\mathrm{ng} / \mathrm{mL})$} & \multicolumn{2}{|c|}{ Grupo 1} & \multicolumn{2}{|c|}{ Grupo 2} & \multicolumn{2}{|c|}{ Grupo 3} & \multirow[b]{2}{*}{$\mathrm{p}$} \\
\hline & $\mathrm{n}^{\mathrm{o}}$ & $\%$ & $\mathrm{n}^{\mathrm{o}}$ & $\%$ & $\mathrm{n}^{\mathrm{o}}$ & $\%$ & \\
\hline$<3,7$ & 3 & $\overline{5,5}$ & $\overline{1}$ & 4,3 & 2 & 6,3 & \\
\hline $3,7-9,57$ & 31 & 56,4 & 16 & 69,6 & 20 & 62,5 & \\
\hline$>9,57$ & 21 & 38,2 & 6 & 26,1 & 10 & 31,3 & \\
\hline Total & 55 & 100,0 & 23 & 100,0 & 32 & 100,0 & $0,956^{*}$ \\
\hline
\end{tabular}

Grupo 1: pacientes em uso de TARV com AZT, Grupo 2: pacientes em uso de TARV sem AZT, Grupo 3: pacientes sem uso de TARV. *estatística calculada à Kruskal Wallis, TARV: terapia antirretroviral. 
TABELA 2 - Distribuição dos 110 pacientes infectados pelo HIV, de acordo com os níveis séricos de vitamina $B 12$.

\begin{tabular}{|c|c|c|c|c|c|c|c|}
\hline \multirow[b]{2}{*}{ Vitamina B12 $(\mathrm{pg} / \mathrm{mL})$} & \multicolumn{2}{|c|}{ Grupo 1} & \multicolumn{2}{|c|}{ Grupo 2} & \multicolumn{2}{|c|}{ Grupo 3} & \\
\hline & $\mathrm{n}^{\mathrm{o}}$ & $\%$ & $\mathrm{n}^{\mathrm{o}}$ & $\%$ & $\mathrm{n}^{\mathrm{o}}$ & $\%$ & $\mathrm{p}$ \\
\hline$<150$ & 23 & 41,8 & 5 & 21,7 & 13 & 40,6 & \\
\hline $150-982$ & 32 & 58,2 & 17 & 73,9 & 19 & 59,4 & \\
\hline$>982$ & 0 & 0,0 & 1 & 4,3 & 0,0 & 0,0 & \\
\hline Total & 55 & 100,0 & 23 & 100,0 & 32 & 100,0 & $0,008^{*}$ \\
\hline
\end{tabular}

Grupo 1: pacientes em uso de TARV com AZT, Grupo 2: pacientes em uso de TARV sem AZT, Grupo 3: pacientes sem uso de TARV. *estatística calculada à Kruskal Wallis, TARV: terapia antirretroviral.

$12 \mathrm{~g} / \mathrm{dL}$, no momento atual, não mostraram diferença estatística significativa na comparação entre eles, sendo a distribuição de G1 igual à de G2 e G3 (p = 0,584).
Os grupos estudados apresentaram diferença estatística significativa, na comparação entre si, no que se refere aos níveis de hemoglobina anteriores à pesquisa $(\mathrm{p}=0,004)$, tanto para valores acima quanto abaixo da normalidade.

A distribuição dos indivíduos quanto às dosagens do VCM, antes e no momento atual deste estudo, está mostrada na Tabela 4. Esses grupos apresentaram diferença estatística significativa quando comparados entre si, sendo que os níveis de VCM foram maiores no G1 comparado ao G2 ( $<<0,05)$ e daquele em relação ao G3 ( $\mathrm{p}<0,001)$, no momento atual.

Quanto ao VCM anterior ao início da pesquisa, os grupos não apresentaram diferença estatística significativa entre eles $(p=0,270)$ quanto aos valores de VCM anterior ao estudo.

TABELA 3 - Distribuição de 110 pacientes infectados pelo HIV, de acordo com os níveis de hemoglobina em dois períodos distintos.

\begin{tabular}{|c|c|c|c|c|c|c|c|c|}
\hline & & \multicolumn{2}{|c|}{ Grupo 1} & \multicolumn{2}{|c|}{ Grupo 2} & \multicolumn{2}{|c|}{ Grupo 3} & \multirow[b]{2}{*}{$\mathrm{p}$} \\
\hline & & $\overline{\mathrm{n}^{\circ}}$ & $\%$ & $\overline{\mathrm{n}^{\circ}}$ & $\%$ & $\overline{\mathrm{n}^{\circ}}$ & $\%$ & \\
\hline \multirow{3}{*}{ Hemoglobina atual (g/dL) } & $<12$ & 10 & 18,2 & 4 & 17,4 & 1 & 3,1 & \\
\hline & $>12$ & 45 & 81,8 & 19 & 82,6 & 31 & 96,9 & \\
\hline & total & 55 & 100,0 & 23 & 100,0 & 32 & 100,0 & $0,584^{*}$ \\
\hline \multirow{3}{*}{ Hemoglobina anterior $(\mathrm{g} / \mathrm{dL})$} & $<12$ & 15 & 28,3 & 5 & 21,7 & 1 & 4,0 & \\
\hline & $>12$ & 38 & 71,7 & 18 & 78,3 & 24 & 96,0 & \\
\hline & total & 53 & 100,0 & 23 & 100,0 & 25 & 100,0 & $0,004^{*}$ \\
\hline
\end{tabular}

Grupo 1: pacientes em uso de TARV com AZT, Grupo 2: pacientes em uso de TARV sem AZT, Grupo 3: pacientes sem uso de TARV. *estatística calculada à Kruskal Wallis, TARV: terapia antirretroviral.

TABELA 4 - Distribuição de 102 pacientes infectados pelo HIV, de acordo com o volume corpuscular médio em dois períodos distintos.

\begin{tabular}{|c|c|c|c|c|c|c|c|c|}
\hline & & \multicolumn{2}{|c|}{ Grupo 1} & \multicolumn{2}{|c|}{ Grupo 2} & \multicolumn{2}{|c|}{ Grupo 3} & \multirow[b]{2}{*}{$\mathrm{p}$} \\
\hline & & $\overline{\mathrm{n}^{\mathrm{o}}}$ & $\%$ & $\overline{\mathrm{n}^{\circ}}$ & $\%$ & $\mathrm{n}^{\mathrm{o}}$ & $\%$ & \\
\hline \multirow{3}{*}{ VCM Atual (fl) } & $<100$ & 21 & 40,4 & 13 & 59,1 & 28 & 100 & \\
\hline & $\geq 100$ & 31 & 59,6 & 9 & 40,9 & 0 & 0,0 & \\
\hline & total & 52 & 100,0 & 22 & 100,0 & 28 & 100,0 & $<0,05^{*}$ \\
\hline \multirow{3}{*}{ VCM Anterior (fl) } & $<100$ & 42 & 79,2 & 13 & 56,5 & 25 & 100 & \\
\hline & $\geq 100$ & 11 & 20,8 & 10 & 43,5 & 0,0 & 0,0 & \\
\hline & total & 53 & 100,0 & 23 & 100 & 25 & 100,0 & $0,270^{*}$ \\
\hline
\end{tabular}

VCM: volume corpuscular médio, Grupo 1: pacientes em uso de TARV com AZT, Grupo 2: pacientes em uso de TARV sem AZT, Grupo 3: pacientes sem uso de TARV. * estatística calculada à Kruskal Wallis, TARV: terapia antirretroviral.

\section{DISCUSSÃO}

No presente estudo, a variação das idades dos indivíduos em cada grupo apresentou-se na faixa etária de 30 a 55 anos, dados esses semelhantes aos descritos no Boletim Epidemiológico de DST e AIDS do Ministério da Saúde do Brasil de 2007 e segundo os dados doSINAN (Sistema de Informação de Agravos de Notificação) da Secretaria de Saúde do Município de Maringá-PR, Setor de Vigilância em Saúde ${ }^{1,2}$.

Os resultados da presente casuística demonstraram homogeneidade entre os grupos, quanto ao gênero, com razão entre homens e mulheres de 1,1:1. No Brasil, a partir de 2002, essa relação tem sido de 1,5:1, dados esses que demonstram a característica atual de feminização da epidemia de AIDS no país ${ }^{1}$.

Os pacientes com AIDS se beneficiam, atualmente, do tratamento $\mathrm{ARV}$, que deve ter início quando há progressão da infecção pelo HIV, evoluindo com manifestações clínicas e/ou laboratoriais da AIDS $^{14}$.
Para os pacientes em uso de TARV é esperada maior sobrevida ${ }^{15}$, o que na casuística apresentada foi observada nos grupos 1 e 2, ou seja, naqueles que faziam tratamento medicamentoso e que, também, tinham tempo de diagnóstico da infecção pelo HIV muito maior do que no grupo que ainda não havia indicação de iniciar o tratamento (G3). Isso poderia ser explicado, porém, pelo fato de que os pacientes sem indicação de TARV tiveram seu diagnóstico realizado há menos tempo, média de três anos. Importante ressaltar que a queda na contagem de linfócitos T CD4+ é critério de indicação de tratamento em indivíduos assintomáticos e demonstra evolução da doença ${ }^{14}$.

O tempo médio de tratamento com o uso de TARV dos indivíduos do grupo que incluía o AZT (G1) em seu esquema foi de três anos e meio, enquanto no grupo de pacientes em uso de TARV sem AZT (G2), foi de sete anos em média. O AZT, além de ter sido a primeira droga disponível para uso clínico em doentes com AIDS, foi, também, utilizado por anos como monoterapia, dupla terapia e faz parte dos esquemas tríplices de escolha para início da TARV 
até os dias atuais ${ }^{14}$. Dessa forma, os doentes do grupo sem AZT, provavelmente, tiveram essa droga substituída por alguma razão ao longo do tempo de tratamento, seja por intolerância, toxicidade, resistência, entre outras ${ }^{14}$.

No presente estudo, 69,1\% do total de indivíduos apresentavam contagens de linfócitos T CD4+ elevadas, na faixa acima de 350 células/ $\mathrm{mm}^{3}$, não havendo diferença entre os três grupos. Aqueles indivíduos em uso de TARV, com ou sem AZT, apresentam provável elevação da contagem linfócitos T CD4+ devido ao uso correto e prolongado da terapia, com recuperação parcial da contagem dessas células. Praticamente, o mesmo percentual dos doentes (68,8\%), apresentava carga viral plasmática do HIV abaixo do limite mínimo de detecção, ou seja, boa resposta virológica ao TARV, para os grupos com tratamento.

O estágio crônico e tardio da infecção pelo HIV, quando acompanhado por nutrição inadequada e má absorção de micronutrientes, pode levar à deficiência calórico-protéica e distúrbios metabólicos, destacando-se a deficiência do ácido fólico e de vitamina B12. A deficiência de vitamina B12 ocorre em 10\% a 39\% dos infectados pelo HIV, e a deficiência de folato, por má-absorção do ácido fólico, em $57 \%$ a $64 \%$ dos casos ${ }^{16}$.

No presente trabalho, concentrações de ácido fólico foram dosadas em portadores de HIV, cuja análise dos resultados não demonstrou diferença estatística na proporção de indivíduos com níveis baixos, normais ou altos, entre os grupos, conforme o uso ou não de ARV, mesmo com a utilização de esquemas contendo AZT. Porém, a maioria dos pacientes apresentava os níveis séricos de ácido fólico dentro do limite da normalidade e, apenas a minoria de cada grupo apresentava deficiência de folato, que não se correlacionava com o uso de TARV.

Com relação às dosagens séricas de vitamina $\mathrm{B} 12$, os dados encontrados no presente estudo, mostraram que a maioria dos indivíduos apresentava níveis normais da vitamina, sendo, principalmente, os do grupo que usava TARV sem AZT. Nos grupos estudados, boa parte dos indivíduos, mais de $40 \%$, apresentava-se com deficiência de vitamina $\mathrm{B} 12$, nos grupos em uso de TARV incluindo AZT e naqueles sem uso de TARV e, que no último caso, tinham menos tempo de diagnóstico da infecção pelo HIV. Apesar do uso prolongado de TARV, no presente trabalho, incluindo AZT, não foi possível estabelecer essas medicações como causa de deficiência de vitamina B12, bem como não pode ser relacionada à macrocitose ou à anemia.

Remacha cols observaram que a baixa concentração da vitamina B12 e de folato ocorreram em $20 \%$ e $10 \%$, respectivamente, dos pacientes infectados pelo HIV. Esses autores observaram, ainda, que os níveis de ácido fólico dos pacientes que estavam em uso de TARV não apresentaram diferença estatística se comparados ao grupo sem TARV, resultados semelhantes aos mostrados no presente estudo. Destacaram, também, que a baixa concentração de vitamina B12 e a deficiência de folato poderiam ser indicador de progressão da doença em indivíduos infectados pelo $\mathrm{HIV}^{17}$.

$\mathrm{O}$ estudo realizado por Malavazi cols ${ }^{18}$ demonstrou que a determinação, tanto de folato, quanto de vitamina B12, encontravamse dentro da normalidade, porém próximos de seus limites inferiores, independentemente de usarem ou não TARV. Eles citam, ainda, que os valores diminuídos de vitamina B12 detectados anteriormente ocorriam em qualquer estágio da infecção pelo HIV, sendo eles causados principalmente por anormalidades das proteínas ligantes da vitamina B12, como a transcobalamina ${ }^{16}$.
Uma das complicações mais frequentes do uso da TARV é a anemia detectada laboratorialmente. Essa anemia é usualmente normocrômica e normocítica, apesar de haver evidências de macrocitose em indivíduos em uso de TARV, principalmente, com zidovudina e estavudina. O AZT foi correlacionado com a ocorrência de anemia em indivíduos infectados pelo $\mathrm{HIV}^{4}$.

Os valores de hemoglobina obtidos no presente trabalho não foram diferentes entre os grupos e não foi possível a caracterização de anemia. No entanto, $17,9 \%$ da população em tratamento com TARV apresentavam valores de hemoglobina abaixo do valor normal considerado na pesquisa ( $12 \mathrm{~g} / \mathrm{dL})$, em comparação a $3,1 \%$ dos que não estavam em tratamento no momento atual. Clinicamente, esses pacientes encontravam-se assintomáticos, inclusive aqueles em uso de TARV.

A anemia, entretanto, é comum em indivíduos infectados pelo HIV, ocorrendo em aproximadamente $30 \%$ dos assintomáticos e $60 \%$ a $70 \%$ daqueles com AIDS, e está relacionada com a qualidade de vida e sobrevida dos mesmos ${ }^{15}$. Semba cols ${ }^{5}$, Berhane ${ }^{6}$ e Mocroft cols ${ }^{19}$ demonstraram que o uso prolongado da medicação foi um fator de resolução da anemia, após 6 meses de seu uso e com significante proteção contra a anemia após 12 meses.

No presente estudo, os valores de hemoglobina anteriores à pesquisa demonstraram que os pacientes que já estavam em uso de TARV com AZT se encontravam com níveis menores de hemoglobina, quando comparados ao grupo com os outros grupos. Além disso, em todos os grupos notou-se discreto aumento dos níveis de hemoglobina ao se comparar as dosagens anteriores à pesquisa e as atuais.

Alguns autores ${ }^{11,18}$ citam o uso da TARV, especialmente do AZT, como causa da macrocitose, que ocorre por diminuição do tempo do ciclo de divisão da célula, produzindo células ampliadas ${ }^{8}$. No presente estudo, indivíduos em uso de TARV com AZT apresentaram valores de VCM significativamente maiores do que os indivíduos em uso de TARV sem AZT e, inclusive, quando comparados àqueles infectados pelo HIV sem TARV. Sabe-se que outros antirretrovirais, principalmente, a estavudina, podem, também, causar macrocitose, o que não foi diferenciado no presente estudo ${ }^{12}$.

Genné cols ${ }^{12}$, ao estudarem causas de macrocitose em pacientes infectados pelo HIV não tratados com AZT, concluíram que os níveis de ácido fólico e vitamina B12 não se correlacionavam com a macrocitose, dados que foram semelhantes aos encontrados no presente estudo.

É interessante chamar atenção para um dado do presente estudo, ao se avaliar as dosagens de hemoglobina sérica e macrocitose, prévias e no momento do estudo. Enquanto houve redução do número de pacientes com anemia durante o estudo, em sentido inverso, houve aumento do número de indivíduos que apresentavam macrocitose no mesmo período, quando comparado com os dados anteriores ao estudo. Apesar da ocorrência de macrocitose na maioria dos indivíduos em uso de TARV com AZT e quase metade daqueles com TARV sem AZT, não foi possível relacioná-la ao uso das medicações.

Assim sendo, embora o uso de ARV, especialmente o AZT e o $\mathrm{d} 4 \mathrm{~T}$, sugira ser fator causador de macrocitose, isso não foi confirmado nesse estudo, e esta macrocitose observada pode ter ocorrido por outras etiologias não definidas, apesar do uso de TARV.

Certamente, outros estudos devem ser realizados na tentativa de encontrar as causas de macrocitose em doentes com AIDS ou relacioná-la às deficiências de vitamina $\mathrm{B} 12$ e/ou ácido fólico, que, eventualmente, possam atuar como complicadores de anemia ou macrocitose na vigência de terapia antirretroviral. 


\section{AGRADECIMENTOS}

Agradeço ao Programa deDST/AIDS do município de Maringá-PR e ao Laboratório de Análises Clínicas do Município de Maringá-PR.

\section{CONFLITO DE INTERESSE}

Os autores declaram não haver nenhum tipo de conflito de interesse no desenvolvimento do estudo.

\section{REFERÊNCIAS}

1. Boletim Epidemiológico AIDS [Intenet]. Boletim $n^{\circ} 1$, ano V, julho a dezembro de 2007, janeiro a julho de 2008. [Acessado 10 jan 2010]. Disponível em www. aids.gov.br/.

2. Secretaria de Vigilância em Saúde [Internet]. Banco de dados do Município de Maringá-PR. Perfil Epidemiológico de AIDS em Maringá nos anos de 2007 e 2008. [Acessado 06 out 2008]. Disponível em saude.maringa.gov.br/.

3. Silva EB, Grotto HZ, Vilela MM. Aspectos clínicos e o hemograma em crianças expostas ao HIV-1:comparação entre pacientes infectados e soro-reversores. J Pediatr (Rio J) 2001; 77:503-511.

4. Montessori V, Press N, Harris M, Akagi L, Montaner JS. Adverse effects of antiretroviral therapy for HIV infection. CMAJ 2004; 170:229-238.

5. Semba RD, Gray GE. Pathogenesis of anemia during human immunodeficency virus infection. J Investig Med 2001; 49:225-239.

6. Berhane K, Karim R, Cohen MH, Masri-Lavine L, Young M, Anastos K, et al Impact of active antiretroviral therapy on anemia and relationship between anemia and survival in HIV infected women. J Acquir Immune Defic Syndr 2004;37:1245-1252.

7. Weiss G. Pathogenesis and treatment of anaemia of chronic disease. Blood Rev 2002; 16:87-96.

8. Romanelli F, Empey K, Pomeroy C. Macrocytosis as an indicator of medication zidovudine adherence in patientes with HIV infection. AIDS Patient Care STDS 2002; 16:405-411.

9. Semba R, Tang A. Micronutrients and pathogenesis of AIDS infection. BRJ Nutricion 1999; 81:181-199.

10. Jennings HJ, Romanelli F. Zidovudine-induced macrocytosis as a marker of antiretroviral adherence in HIV-positive patients. J Am Pharm Assoc 1999; 39:150.

11. Richman DD, Fischl MA, Grieco MH, Gottlieb MS, Volberding PA, Laskin OL, et al. The toxicity of azidothymidine (AZT) in the treatment of patients with AIDS and AIDS-related complex. A double-blind, placebo-controlled trial. N Engl J Med 1987; 317:192-197.

12. Geené D, Sudre P, Anwar D, Goehring C, Saaïdia A, Hirschel B. Causes of macrocytosis in HIV-infected Patients not Treated with Zidovudine. J Infect 2000; 40:160-163.

13. Magalhaes-Silvia MM. Síndromes mielodisplásticas: diagnóstico de exclusão. Rev Bras Hematol Hemoter 2006; 28:175-177.

14. Ministério da Saúde. Programa Nacional de DST e AIDS. Recomendações para terapia anti retroviral em adultos e adolescentes infectados pelo HIV. Brasília: Secretaria de Vigilância em Saúde; 2006.

15. Doukas, MA. Human immunodeficiency virus associated anemia. Med Clin North Am 1992;76:699-709.

16. Remacha AF, Cadafalch J. Colabamin deficiency in patients infected with the human immunodeficiency virus. Semin Hematol 1999; 36:75-87.

17. Remacha AF, Cadafalch J, Sardà P, Barceló M, Fuster M. Vitamin B metabolism in HIV-infected patients in the age of highly active antiretroviral therapy: role of homocysteine in assessing vitamin B12 status. Am J Clin Nutr 2003; 77:420-424.

18. Malavazi I, Abrão EP, Mikawa AY, Tagliavini SA, da Costa PI. Evaluation of the polymorphisms in methylenetetrahydrofolate reductase gene and the levels of folate and B12 in HIV infected patients under antiretroviral therapy. Rev Soc Bras Med Trop 2004; 37:469-475.

19. Mocroft A, Kirk O, Barton SE, Dietrich E, Proenca R, Colebunders R, et al Anaemia is an independent predictive marker for clinical prognosis in HIVinfected patients from across Europe. AIDS 1999; 13:943-950. 